\title{
Mobile Spine Osseous Sarcoma: Descriptive Epidemiological Analysis Based on a National Population-Based Study
}

\author{
Adel Ebrahimpour, MD ${ }^{1,2}$; Mohammadreza Chehrassan, $\mathrm{MD}^{1,2^{*}}$; Mehrdad Sadighi, MD ${ }^{1,2}$; Mehdi Azizmohammad Looha, MSc

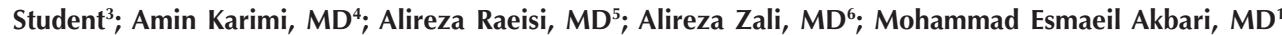 \\ 'Cancer Research Center, Shahid Beheshti University of Medical Sciences, Tehran, Iran \\ ${ }^{2}$ Department of Orthopedic Surgery, Shohadaye Tajrish Hospital, Shahid Beheshti University of Medical Sciences, Tehran, Iran \\ ${ }^{3}$ Department of biostatics, Faculty of paramedical sciences, Shahid Beheshti University of Medical Sciences, Tehran, Iran \\ ${ }^{4}$ Department of Orthopedic Surgery, Taleghani Hospital, Shahid Beheshti University of Medical Sciences, Tehran, Iran \\ ${ }^{5}$ Shiraz University of Medical Sciences, Shiraz, Iran \\ ${ }^{6}$ Functional Neurosurgery Research Center, Shahid Beheshti University of Medical Sciences, Tehran, Iran
}

\begin{abstract}
Background: Primary osseous sarcomas of the mobile spine are rare bony tumors. Ewing sarcoma, chondrosarcoma, chordoma and osteosarcoma constitute the majority of primary bone sarcomas of the spine; however, other rare sarcoma tumors may also affect the spine. In order to perform an epidemiological study of theses tumors, national registries may help to evaluate data for populations with similar characteristics.

Methods: A population-based study was designed based on data from the Iran National Cancer Registry (INCR). All morphology codes (M-Code) of primary osseous sarcomas of the mobile spine (C-code 41.2) were derived and analyzed.

Results: Among 186 patients with primary osseous sarcomas of the mobile spine, $67.2 \%$ were men and $32.8 \%$ were women. The median (IQR) age was $37.0(20.0-59.0)$ years and the age-standardized incidence rate (ASIR) was 0.37 per million. The majority of cases of Ewing sarcoma (29.5\%) were observed in the age group 20-25 years. Among male patients with chondrosarcoma, the median age was 39.0 (30.0-50.0), while females showed a median age of 56.0 (50.0-59.0). The median age of patients with chordoma was 54.0 (47.0-63.0) years. The crude incidence rate of mobile spine osteosarcoma was 0.04 per million.

Conclusion: Ewing sarcoma was the most frequent primary osseous sarcoma of the mobile spine. A male predilection was observed among all major sarcomas of the mobile spine. Ewing sarcoma in Iran affects the mobile spine in slightly older ages compared to other studies. Myxoid chondrosarcoma is the most frequent subtype of the mobile spine chondrosarcoma. Chordoma affects male in older ages compared to females. In contrast with other studies which showed a bimodal distribution of osteosarcoma of the spine including young adult and older age groups, $86 \%$ of cases in Iran were in the age group of 10-40 years.

Keywords: Osseous sarcoma, Primary tumor, Spine sarcoma, Vertebral sarcoma

Cite this article as: Ebrahimpour A, Chehrassan M, Sadighi M, Azizmohammad Looha M, Karimi A, Raeisi A, et al. Mobile Spine Osseous Sarcoma: Descriptive Epidemiological Analysis Based on a National Population-based Study. Arch Iran Med. 2021;24(8):629-635. doi: 10.34172/aim.2021.89
\end{abstract}

Received: February 13, 2020, Accepted: March 3, 2021, ePublished: August 1, 2021

\section{Introduction}

Primary osseous sarcomas of the mobile spine, including cervical, thoracic and lumbar spine, are rare and represent $2.6 \%$ to $13 \%$ of all bone tumors. ${ }^{1,2}$ Due to the rarity of theses neoplasms, epidemiologic trends of primary sarcoma of the spine need large number of cases which can be achieved only through national or regional cancer registries.

Chondrosarcoma, osteosarcoma, chordoma and Ewing sarcoma constitute the majority of primary bone sarcomas of the spine; however other less frequent sarcoma tumors may also affect the spine. Ewing sarcoma and osteosarcoma represents $3.5 \%$ and $3 \%-15 \%$ of primary osseous sarcomas of the bone, respectively, and more frequently affect younger age patients. There are different subtypes of osteosarcoma; however, the majority of these subtypes have not been reported in the spine. Chondrosarcoma accounts for nearly $25 \%$ of primary osseous sarcoma tumors and affects older patients compared to osteosarcoma and Ewing sarcoma. Chordoma makes up only $6 \%$ of all bone sarcomas and affect solely the spinal column; however, the majority of cases affect non-mobile spine (sacro-coccyx) and only $15 \%$ of patients have mobile spine involvement. ${ }^{3-5}$

National studies help to analyze the patients of relatively similar race, ethnicity and genetic characteristics. Different national registries like the Surveillance, Epidemiology, and End Results (SEER) Registry, National Cancer Database (NCDB), Scandinavian sarcoma group and similar registries in other countries have been stablished in order to recruit large numbers of patients for epidemiological studies of different cancers among patients of nearly similar race and ethnicity characteristics. ${ }^{6-8}$ To the best of 
our knowledge, in the Middle-East and more precisely in Iran, there is no study on the epidemiological aspects of primary osseous sarcomas of the mobile spine. Considering the latter, a comprehensive study was designed aiming to evaluate the epidemiologic characteristics of primary osseous sarcoma of the mobile spine in the territory of Iran based on the Iran National Cancer Registry (INCR). The INCR is a preliminary registry of cancers in the territory of Iran based on data provided by the Ministry of Health and Medical Education.

\section{Materials and Methods}

A population-based study was designed based on data from the INCR ${ }^{9,10}$ between March 20, 2008, and March 20, 2015. Using the first revision of the third edition of International Classification of Diseases for Oncology (ICD-O-3), ${ }^{11}$ all Morphology codes (M-Code) of primary osseous sarcoma of the mobile spine (C-code 41.2) were derived from the
INCR and registered (Table 1). Only microscopically and pathologically confirmed cases of primary osseous sarcoma of the mobile spine in the territory of Iran were derived from data registry and enrolled in this study. Data of cases which did not meet the defined standards and cases with incomplete information were excluded from the study; moreover, the incomplete histopathology registration of the patients, lack of histology codes in the INCR and duplicate cases were considered as exclusion criteria and were removed.

Cases with the same first and last name, sex, father's name, age and $\mathrm{M}$-code were identified as duplicate records and were removed. The inclusion criteria were surname, sex, father's name, age and M-code, histology codes on INCR and confirmed pathologic results. Initially, 937 cases with diagnosed tumors of the mobile spine (C-code 41.2) were evaluated, but based on the inclusion and exclusion criteria, only 186 cases met the defined standards and

Table 1. Frequency and Age-Standardized Incidence Rate (ASIR) for Each Morphology Code Based on the Third Version of the ICD-O from 2008 to 2015 in Iran

\begin{tabular}{|c|c|c|c|c|c|c|c|c|}
\hline \multirow{3}{*}{ Combined Group } & \multirow{3}{*}{ M-code } & \multicolumn{3}{|c|}{$2008-2014$} & \multicolumn{4}{|c|}{$2008-2014$} \\
\hline & & \multicolumn{3}{|c|}{$\begin{array}{l}\text { Frequency (ASIR) } \\
\text { For M-Code }\end{array}$} & \multicolumn{4}{|c|}{$\begin{array}{l}\text { Frequency (ASIR) } \\
\text { For Combined Group }\end{array}$} \\
\hline & & Total & Male & Female & Total & Male & Female & $P$ value ${ }^{*}$ \\
\hline Fibrosarcoma & $8810=$ Fibrosarcoma & $4(0.01)$ & $2(0.01)$ & $2(0.01)$ & $4(0.01)$ & $2(0.01)$ & $2(0.01)$ & ---- \\
\hline MFH & $8830=\mathrm{MFH}$ & $3(0.01)$ & $3(0.01)$ & ---- & $3(0.01)$ & $3(0.01)$ & ---- & ---- \\
\hline Ewing Sarcoma & 9260=Ewing sarcoma & $44(0.08)$ & $33(0.11)$ & $11(0.04)$ & $44(0.08)$ & $33(0.11)$ & $11(0.04)$ & 0.001 \\
\hline $\begin{array}{l}\text { Peripheral } \\
\text { neuroectodermal } \\
\text { (PPNET) }\end{array}$ & 9364=PPNET & $3(0.01)$ & $2(0.01)$ & $1(0.00)$ & $3(0.01)$ & $2(0.01)$ & $1(0.00)$ & ---- \\
\hline \multirow{2}{*}{ Osteosarcoma } & 9180=Osteosarcoma & $18(0.03)$ & $12(0.04)$ & $6(0.02)$ & \multirow{2}{*}{$23(0.04)$} & \multirow{2}{*}{$14(0.05)$} & \multirow{2}{*}{$9(0.03)$} & \multirow{2}{*}{0.450} \\
\hline & 9181=Chondroblastic osteosarcoma & $5(0.01)$ & $2(0.01)$ & $3(0.01)$ & & & & \\
\hline \multirow{2}{*}{ Chordoma } & $9370=$ Chordoma & $31(0.07)$ & $18(0.08)$ & $13(0.06)$ & \multirow{2}{*}{$35(0.08)$} & \multirow{2}{*}{$21(0.09)$} & \multirow{2}{*}{$14(0.07)$} & \multirow{2}{*}{0.311} \\
\hline & 9371=Chondroid chordoma & $4(0.01)$ & $3(0.01)$ & $1(0.01)$ & & & & \\
\hline \multirow{4}{*}{ Chondrosarcoma } & 9220=Chondrosarcoma & $33(0.07)$ & $21(0.08)$ & $12(0.06)$ & \multirow{4}{*}{$43(0.09)$} & \multirow{4}{*}{$28(0.11)$} & \multirow{4}{*}{$15(0.07)$} & \multirow{4}{*}{0.066} \\
\hline & 9231=Myxoid chondrosarcoma & $2(0.00)$ & ----- & $2(0.01)$ & & & & \\
\hline & 9240=Mesenchymal chondrosarcoma & $6(0.01)$ & $5(0.02)$ & $1(0.00)$ & & & & \\
\hline & 9243=Dedifferentiated chondrosarcoma & $2(0.00)$ & $2(0.01)$ & ---- & & & & \\
\hline \multirow{12}{*}{ Others } & $8800=$ Non classified sarcoma NOS & $13(0.03)$ & $9(0.03)$ & $4(0.02)$ & \multirow{12}{*}{$31(0.06)$} & \multirow{12}{*}{$22(0.08)$} & \multirow{12}{*}{$9(0.04)$} & \multirow{12}{*}{0.029} \\
\hline & $8801=$ Spindle cell sarcoma & $5(0.01)$ & $3(0.01)$ & $2(0.01)$ & & & & \\
\hline & $8803=$ Small cell sarcoma & $3(0.00)$ & $3(0.01)$ & $0(0.00)$ & & & & \\
\hline & 8804=Epithelioid sarcoma & $1(0.00)$ & $0(0.00)$ & $1(0.00)$ & & & & \\
\hline & $8805=$ Undifferentiated sarcoma & $1(0.00)$ & $1(0.01)$ & $0(0.00)$ & & & & \\
\hline & 8832=Dermatofibrosarcoma, NOS & $1(0.00)$ & $1(0.00)$ & $0(0.00)$ & & & & \\
\hline & 8850=Atypical lipoma & $1(0.00)$ & $1(0.00)$ & $0(0.00)$ & & & & \\
\hline & 8851=Liposarcoma well differentiated & $1(0.00)$ & $1(0.00)$ & $0(0.00)$ & & & & \\
\hline & 8852=Myxoid liposarcoma & $1(0.00)$ & $1(0.00)$ & $0(0.00)$ & & & & \\
\hline & $8890=$ leiomyosarcoma & $1(0.00)$ & $1(0.00)$ & $0(0.00)$ & & & & \\
\hline & 9040=Synovial sarcoma & $2(0.00)$ & $0(0.00)$ & $2(0.01)$ & & & & \\
\hline & 9050=Mesothelioma & $1(0.00)$ & $1(0.00)$ & $0(0.00)$ & & & & \\
\hline Total & Total & $186(0.37)$ & $125(0.48)$ & $61(0.26)$ & $186(0.37)$ & $125(0.48)$ & $61(0.26)$ & $<0.001$ \\
\hline
\end{tabular}

* The $P$ value assuming that there is a significant difference between the number of cancers identified in men and women. The $P$ values are for exact binomial proportion test to evaluate the difference between frequency in men and women. $P$ values were not reported for groups with a sample size below 20. 
were selected for this study.

Variables such as age, sex and type of the tumor were derived and registered. All M-codes were evaluated separately and then in order to simplify, the results all subtypes of the same tumor families were combined under the name of the main tumor family group and reevaluated. Patients were divided in different age groups. The crude incidence rate and age-specific incidence rate were calculated for each age group and sex. The trend of age-standardized incidence rate (ASIR) from March 2008 to March 2015 was calculated for each gender.

In the statistical analysis, descriptive statistics, median [interquartile range (IQR): first quartile (Q1) - third quartile (Q3)] for continuous variables (including morphology groups and their combined groups) and frequency (percentage (for categorical variable were used to summarize the information. The exact binominal proportion test was performed to compare the true proportion of two groups. All statistical analyses were performed using SPSS (version 26) and R version 3.6.0. $P$ value $<0.05$ was considered to be statistically significant.

\section{Results}

Among the 186 cases, 44 cases were Ewing sarcoma, 43 cases were chondrosarcoma, 35 cases were chordoma, 23 cases were osteosarcoma, 4 cases were fibrosarcoma, 3 cases were malignant fibrous histiocytoma (MFH), 3 cases were peripheral primitive neuroectodermal (PPNET) and 31 cases were less frequent primary osseous sarcomas and non-classified sarcomas (Table 1$)$.

Considering all sarcomas, $125(67.2 \%)$ of cases were men and $61(32.8 \%)$ were women $(P$ value $<0.001)$. All major sarcomas of the spine, including Ewing sarcoma, Chondrosarcoma, Chordoma and Osteosarcoma showed a male predilection; however, a significant statistical relation between sex and tumor type was observed solely among patients with Ewing sarcoma ( $P$ value:0.001) (Table 1).

The median age of the primary osseous sarcomas of the mobile spine was 34.0 (Q1-Q3: 20.0-51.0) and 40.0 (Q1-
Q3: 24.0-59.0) in men and women, respectively, and 37.0 (Q1-Q3: 20.0-59.0) regardless of gender (Table 2). The ASIR during 2008 to 2015 by gender is shown in Figure 1. The ASIR for primary osseous sarcoma of the mobile spine was 0.37 per million person-years.

For the 44 cases of Ewing sarcoma, the male-to-female ratio was 3:1 (33 men and 11 women) $(P$ value $=0.001)$. The ASIR of Ewing sarcoma in mobile spine was 0.08 in one million persons per year. The median age of Ewing sarcoma was 22.0 years (Q1-Q3: 15.0-28.0 years). In male patients with Ewing sarcoma, the median age was 22.0 (Q1-Q3: 15.0-28.0), while females showed a median age of 20.0 (Q1-Q3: 15.0-27.0). Despite this difference, using the exact Mann-Whitney test, the median age of patients with Ewing sarcoma was not affected by gender $(P$ value $=0.654)($ Table 2$)$. The majority of cases with Ewing sarcoma of the mobile spine were observed in the age group 20-25 years (29.5\%) followed by the age group 15-19 years (20.4\%). Any relation between age group and the sex could not be investigated due to sparse data. However, the difference in new cases of Ewing sarcoma was significant between men and women $(P$ value $<0.05)$. Despite similar pathological, clinical, and radiological characteristics of PPNET and Ewing sarcoma, they were analyzed separately and PPNET was considered as a rare tumor.

Among 43 patients with mobile spine chondrosarcoma, 33 cases $(76 \%)$ were listed as chondrosarcoma, not otherwise specified (M-code: 9220), 6 cases (13.9\%) were Mesenchymal Chondrosarcoma (M-code: 9240), 2 cases (4.6\%) were Myxoid chondrosarcoma (M-code: 9231$)$ and 2 cases (4.6\%) were dedifferentiated chondrosarcoma (M-code: 9243) (Table 1). In the 43 cases of chondrosarcoma, 28 (65.2\%) were men and 15 (34.8\%) were women without any statistical significance $(P$ value $=0.066)$. The ASIR of chondrosarcoma of the mobile spine was 0.08 per million person-years. The median age of patients with chondrosarcoma was 47.00 (IQR: 33.056.0). Among male patients, the median age was 39.0 (30.0 - 50.0), while females showed a median age of 56.0

Table 2. Median (Q1 - Q3) Age (Years) for Patients from 2008 to 2015 in Iran

\begin{tabular}{|c|c|c|c|c|}
\hline \multirow{2}{*}{ Combined Group } & \multicolumn{4}{|c|}{ Median (Q1 - Q3) } \\
\hline & Total & Male & Female & Exact $P$ Value* \\
\hline Fibrosarcoma & $30.50(3.8,57.3)$ & $30.50(3.0,--)$ & $30.50(0.0,--)$ & ---- \\
\hline MFH & $46.00(28.0,--)$ & $46.00(28.0,--)$ & ----- & ---- \\
\hline Ewing sarcoma & $22.00(15.0,28.0)$ & $22.00(15.0,28.0)$ & $20.00(15.0,27.0)$ & 0.654 \\
\hline PPNET & $11.00(7.0,--)$ & $9.00(7.0,--)$ & $21.00(21.0,21.0)$ & --- \\
\hline Osteosarcoma & $25.00(18.0,37.0)$ & $22.50(16.5,37.25)$ & $31.00(19.0,37.0)$ & 0.526 \\
\hline Chordoma & $54.00(47.0,63.0)$ & $53.00(41.0,59.0)$ & $59.50(50,68.3)$ & 0.120 \\
\hline Chondrosarcoma & $47.00(33.0,56.0)$ & $39.00(30.0,50.0)$ & $56.00(50.0,59.0)$ & 0.002 \\
\hline Others & $38.00(23.0,59.0)$ & $33.00(20.0,47.0)$ & $59.00(32.5,62.0)$ & 0.115 \\
\hline Total & $37.00(20.0-59.0)$ & $34.00(20.0,51.0)$ & $40.00(24.0,59.0)$ & 0.012 \\
\hline
\end{tabular}

* The exact $P$-value assuming that there is a significant difference between the mean rank age at the diagnosis of the cancer identified in men and women using Man-Whitney $U$ test. 
(50.0-59.0). The relation between median age and sex was significant with a higher value of median age for women compared to men $(P$ value $=0.002)$. Thirty cases of mobile spine chondrosarcoma $(69.7 \%)$ were between the ages of 30 and 60 (18 men and 12 women).

Chordoma contained two subgroups of chordoma and chondroid chordoma with 31 cases (18 men, 13 women) and 4 cases ( 3 men, 1 woman), respectively. The difference in the number of new cases was not significant between men and women in each subgroup $(P$ value $>0.05)$ (Table 1). The ASIR of mobile spine chordoma was 0.07 per million person-years. The median age of patients with chordoma was 54.0 (47.0-63.0) while men showed a higher median age of $53.0(41.0-59.0)$ years in comparison to women with 59.5 (50.0-68.3) years (Table 2). Most of the new cases occurred in the age group of 45-65 years; however, the relationship between age groups and sex was not statistically verifiable ( $P$ value $>0.05)$.

Out of 23 patients with osteosarcoma, 18 cases were osteosarcoma, not otherwise specified (M-code: 9180$)$ and 5 cases were chondroblastic (M-code: 9181) osteosarcoma (Table 1). Among these 23 patients, 14 were men and 9 were women without any statistical significance $(P$ value $=0.450)$. The crude incidence rate of mobile spine osteosarcoma was 0.04 per million person-years. The median age of osteosarcoma for men was 22.5 (Q1-Q3: 16.5-37.3) which was not significantly lower compared to the median age of women with the value of 31.0 (Q1-Q3:
19.0-37.0) (Table 2). The majority of new cases occurred in the age group of 10-40 years with 20 cases (12 men and 8 women); the relation between age groups and sex could not be evaluated for osteosarcoma (Table 3).

\section{Discussion}

Spine sarcoma includes a variety of malignant tumors the majority of which are rare. Except chordoma, all major osseous sarcomas frequently affect long bones and rarely involve the spine. More frequent sarcomas of the spine include Ewing sarcoma, chondrosarcoma, chordoma and osteosarcoma. Some national registries show a significant correlation between tumor type and race among patients with particular sarcomas. ${ }^{3,7,12}$ Despite different multiinstitutional and national registry studies on demographic and epidemiologic outcome of spine sarcoma in developed countries, there are few works available in other part of the world with different ethnic and race characteristics. To the best of our knowledge, this is the first epidemiological study of spine sarcomas based on a national database registry in a Middle Eastern country with a population of nearly similar racial and ethnic characteristics.

According to the INCR, among patients with primary osseous sarcoma of the mobile spine, $23.6 \%$ had Ewing sarcoma, $23.1 \%$ had chondrosarcoma, $18.8 \%$ had chordoma, $12.3 \%$ had osteosarcoma and $22.2 \%$ had other rare tumors. In a study by Kerr et $\mathrm{al}^{7}$ based on the NCDB, from 2004 to 2015, out of 1011 primary osseous sarcomas

Table 3. Number of New Cases (Age-Specific Incidence Rate/Per Million Person-Years) for Osteosarcoma, Chondrosarcoma, Ewing Sarcoma, and Chordoma during 2008-2015

\begin{tabular}{|c|c|c|c|c|c|c|c|c|c|c|c|c|}
\hline \multirow{2}{*}{ Age } & \multicolumn{3}{|c|}{ Osteosarcoma } & \multicolumn{3}{|c|}{ Chondrosarcoma } & \multicolumn{3}{|c|}{ Ewing Sarcoma } & \multicolumn{3}{|c|}{ Chordoma } \\
\hline & Total & Male & Female & Total & Male & Female & Total & Male & Female & Total & Male & Female \\
\hline $0-4$ & $0(0.00)$ & $0(0.00)$ & $0(0.00)$ & $1(0.02)$ & $1(0.04)$ & $0(0.00)$ & $3(0.07)$ & $3(0.13)$ & $0(0.00)$ & $0(0.00)$ & $0(0.00)$ & $0(0.00)$ \\
\hline $5-9$ & $0(0.00)$ & $0(0.00)$ & $0(0.00)$ & $0(0.00)$ & $0(0.00)$ & $0(0.00)$ & $0(0.00)$ & $0(0.00)$ & $0(0.00)$ & $0(0.00)$ & $0(0.00)$ & $0(0.00)$ \\
\hline $10-14$ & $2(0.05)$ & $2(0.10)$ & $0(0.00)$ & $0(0.00)$ & $0(0.00)$ & $0(0.00)$ & $4(0.10)$ & $3(0.14)$ & $1(0.05)$ & $1(0.02)$ & $1(0.05)$ & $0(0.00)$ \\
\hline $15-19$ & $5(0.11)$ & $3(0.13)$ & $2(0.09)$ & $2(0.04)$ & $2(0.08)$ & $0(0.00)$ & $9(0.19)$ & $6(0.25)$ & $3(0.13)$ & $0(0.00)$ & $0(0.00)$ & $0(0.00)$ \\
\hline $20-24$ & $4(0.07)$ & $2(0.07)$ & $2(0.07)$ & $1(0.02)$ & $1(0.04)$ & $0(0.00)$ & $13(0.23)$ & $9(0.32)$ & $4(0.14)$ & $1(0.02)$ & $1(0.04)$ & $0(0.00)$ \\
\hline $25-29$ & $2(0.03)$ & $2(0.07)$ & $0(0.00)$ & $2(0.03)$ & $2(0.07)$ & $0(0.00)$ & $6(0.10)$ & $5(0.17)$ & $1(0.03)$ & $2(0.03)$ & $2(0.07)$ & $0(0.00)$ \\
\hline $30-34$ & $2(0.04)$ & $0(0.00)$ & $2(0.08)$ & $6(0.12)$ & $5(0.20)$ & $1(0.04)$ & $1(0.02)$ & $0(0.00)$ & $1(0.04)$ & $2(0.04)$ & $0(0.00)$ & $2(0.08)$ \\
\hline $35-39$ & $5(0.13)$ & $3(0.15)$ & $2(0.10)$ & $3(0.08)$ & 3 (0.15) & $0(0.00)$ & $4(0.10)$ & $3(0.15)$ & $1(0.05)$ & $1(0.03)$ & $1(0.05)$ & $0(0.00)$ \\
\hline $40-44$ & $0(0.00)$ & $0(0.00)$ & $0(0.00)$ & $5(0.15)$ & $3(0.17)$ & $2(0.12)$ & $1(0.03)$ & 1 (0.06) & $0(0.00)$ & $1(0.03)$ & $1(0.06)$ & $0(0.00)$ \\
\hline $45-49$ & $0(0.00)$ & $0(0.00)$ & $0(0.00)$ & $3(0.10)$ & $3(0.21)$ & $0(0.00)$ & $0(0.00)$ & $0(0.00)$ & $0(0.00)$ & $4(0.14)$ & $3(0.21)$ & $1(0.07)$ \\
\hline $50-54$ & $1(0.04)$ & $1(0.08)$ & $0(0.00)$ & $6(0.25)$ & $4(0.33)$ & $2(0.17)$ & $1(0.04)$ & $1(0.08)$ & $0(0.00)$ & $6(0.25)$ & $4(0.33)$ & $2(0.17)$ \\
\hline 55-59 & $1(0.05)$ & $1(0.11)$ & $0(0.00)$ & $7(0.38)$ & $0(0.00)$ & $7(0.75)$ & $1(0.05)$ & $1(0.11)$ & $0(0.00)$ & $5(0.27)$ & $3(0.33)$ & $2(0.21)$ \\
\hline $60-64$ & $0(0.00)$ & $0(0.00)$ & $0(0.00)$ & $1(0.08)$ & $0(0.00)$ & $1(0.15)$ & $1(0.08)$ & $1(0.16)$ & $0(0.00)$ & $6(0.45)$ & $3(0.47)$ & $3(0.44)$ \\
\hline $65-69$ & $1(0.10)$ & $0(0.00)$ & $1(0.21)$ & $1(0.10)$ & $1(0.21)$ & $0(0.00)$ & $0(0.00)$ & $0(0.00)$ & $0(0.00)$ & $2(0.21)$ & $0(0.00)$ & $2(0.41)$ \\
\hline $70-74$ & $0(0.00)$ & $0(0.00)$ & $0(0.00)$ & $4(0.51)$ & $3(0.75)$ & $1(0.26)$ & $0(0.00)$ & $0(0.00)$ & $0(0.00)$ & $1(0.13)$ & $0(0.00)$ & $1(0.26)$ \\
\hline $75-79$ & $0(0.00)$ & $0(0.00)$ & $0(0.00)$ & $1(0.16)$ & $0(0.00)$ & $1(0.34)$ & $0(0.00)$ & $0(0.00)$ & $0(0.00)$ & $3(0.49)$ & $2(0.63)$ & $1(0.34)$ \\
\hline 80-85 & $0(0.00)$ & $0(0.00)$ & $0(0.00)$ & $0(0.00)$ & $0(0.00)$ & $0(0.00)$ & $0(0.00)$ & $0(0.00)$ & $0(0.00)$ & $0(0.00)$ & $0(0.00)$ & $0(0.00)$ \\
\hline+85 & $0(0.00)$ & $0(0.00)$ & $0(0.00)$ & $0(0.00)$ & $0(0.00)$ & $0(0.00)$ & $0(0.00)$ & $0(0.00)$ & $0(0.00)$ & $0(0.00)$ & $0(0.00)$ & $0(0.00)$ \\
\hline Total & $23(0.04)$ & $14(0.05)$ & $9(0.03)$ & $43(0.08)$ & $28(0.11)$ & $15(0.06)$ & $44(0.08)^{*}$ & $33(0.12)$ & $11(0.04)$ & $35(0.07)$ & $21(0.08)$ & $14(0.05)$ \\
\hline
\end{tabular}

*The significant difference in number of new cases between men and women.

Numbers in brackets indicate age-specific incidence rate. 
of the whole spine including the sacro-coccygeal zone, 337 cases $(33.3 \%)$ were chordoma, 278 cases $(27.5 \%)$ were Ewing sarcoma, 223 cases (22\%) were chondrosarcoma and 133 cases $(13.1 \%)$ were osteosarcoma. Due to the high tendency of chordoma and Ewing sarcoma to involve the sacro-coccygeal part of the spine and excluding all rare sarcomas of the spine in the latter study, our results show a nearly similar frequency of the main primary sarcomas of the mobile spine.

Regardless of the malignancy type, there is generally a slight male predilection for vertebral tumors. ${ }^{7,13}$ According to our results, there was a statistically significant male predilection for primary osseous sarcomas of the mobile spine which is consistent with other studies.

The median age of the primary osseous sarcomas of the mobile spine was 38 years which means that generally, spine sarcoma more frequently affects the patients during the adult age. There is less predilection for primary spine malignancies among children compared to adults ${ }^{1}$; however, there is a high tendency for malignancy for diagnosed tumors of the spine among children. In one study in 2007 based on a national registry in United Kingdom with 42 years of survey, among patients younger than 18 years, $40 \%$ of primary spine tumors were malignant. ${ }^{14}$

Ewing sarcoma is a family of highly malignant, poorly differentiated tumors composed of small round cells. Ewing sarcoma of the spine and paraspinal region accounts for $3.5 \%$ of all Ewing sarcomas and it affects the sacrum more frequently (55\%) in case of spine involvement. ${ }^{15}$ Racial predilection is observed also among patients affected by Ewing sarcoma, with Caucasians accounting for 93\% of the patients while African-Americans represent only $4 \%{ }^{7,16}$ Spine is a common site for primary appendicular Ewing sarcoma and axial skeleton is the primary site of Ewing sarcomas in only $3 \%$ to $10 \%$ of cases. Considering the childhood and adolescent age range, Ewing sarcoma is the most common nonlymphoproliferative primary osseous sarcoma of the spine..$^{8,13,17,18}$ In Iran, the majority of cases with Ewing sarcoma of the mobile spine were observed among the age group 15 to 25 years and the median age was 22.0, which is slightly higher than other studies. Ewing sarcoma accounts for $23.6 \%$ of all primary osseous sarcomas of the mobile spine derived from the INCR. According to the NCDB, the median age of diagnosis for Ewing sarcoma is 22 years and accounts for $27 \%$ of primary osseous sarcomas of the spine. ${ }^{7}$ The maleto-female ratio in Ewing sarcoma was $62 \%$ to $38 \%$ based on the NCDB while in Iran, Ewing sarcoma affects men three times more than women.

Chondrosarcoma is a malignant tumor with cartilaginous differentiation. ${ }^{19,20}$ Like other sarcomas, there is a racial predilection reported for chondrosarcoma. According to SEER, the Caucasian race represents $89 \%$ of cases in the study. Most patients were diagnosed during the $5^{\text {th }}$ and $6^{\text {th }}$ decades of their life. ${ }^{6}$ The spine represent the primary site for chondrosarcoma in only $3 \%$ to $12 \%$ of cases.
According to the NCDB, chondrosarcoma accounts for $22 \%$ of all spine primary malignancies. ${ }^{7}$ This is consistent with our results in which $23.1 \%$ of primary osseous sarcomas of the mobile spine were chondrosarcomas. The majority of cases with chondrosarcoma of the spine are low-grade, and cases with high grade pathologies remain less frequent. ${ }^{7}$ Chondrosarcoma can present as a primary malignant tumor of the spine or can be a malignant transformation of other benign cartilaginous tumors such as osteochondromas or enchondromas ( $40 \%$ of cases). ${ }^{3}$ In our study, the ASIR of chondrosarcoma of the mobile spine was 0.08 per million person-years. According to the INCR, the median age of patients with chondrosarcoma was 45.5 years which is slightly lower compared to other studies. Using the SEER Registry from 1973 to 2012, Arshi et $\mathrm{al}^{20}$ reported 973 cases of chondrosarcoma with a mean age of 51.6 years at the time of diagnosis. The median age at diagnosis in our study was higher among female patients (56.0 years) compared to males (39.0 years). Among primary osseous sarcomas of the mobile spine, a significant statistical correlation between median age at diagnosis and sex was only observed among chondrosarcoma patients. Despite a male predilection for chondrosarcoma of the mobile spine $(65.2 \%$ were men and $34.8 \%$ were women), our results showed no statistical significance for these entities which is consistent with the results derived from SEER reports (3:2). ${ }^{13,20}$ Generally, the frequency distribution of chondrosarcoma subtypes of appendicular skeleton include myxoid 5.8\%, mesenchymal $2.1 \%$, dedifferentiated $3.5 \%$, and clear cell $0.5 \%$; however, this may be different for the spine. According to the SEER report for chondrosarcoma of the spine, myxoid (5.8\%), dedifferentiated (3.5\%), and mesenchymal chondrosarcoma $(2.1 \%)$ were subtypes of chondrosarcoma and the rest were simply reported as chondrosarcoma. ${ }^{20,21}$ This is in contrast with the results derived from our series in which $13.9 \%$ were mesenchymal chondrosarcoma, $4.6 \%$ were myxoid chondrosarcoma and $4.6 \%$ were dedifferentiated chondrosarcoma.

Chordoma is a malignant tumor from vestigial remnants of the notochord with particular proclivity to involve the cranial base and the vertebral column. According to the NCDB and other studies, African Americans represent only $3 \%$ (2.2\% to $3.3 \%)$ of chordomas which demonstrates the influence of race on this pathology. However, the racial discrepancy for this tumor remains unclear. ${ }^{7}$ About $50 \%$ of chordomas are localized in the sacrum and $35 \%$ in the clivus, which leaves only $15 \%$ in the mobile spine. ${ }^{13,22}$ Chordoma is a low-grade malignant tumor of the axial skeleton with nearly $3 \%$ metastasis at the time of diagnosis. Considering the whole spine, chordoma is the most common primary osseous sarcoma of the spine (37\%); however, excluding the sacrococcygeal region, the mobile spine contains only a smaller percent. ${ }^{7}$ According to our results, $18.8 \%$ of all primary osseous sarcomas of the mobile spine were chordomas which may be consistent with other reports. Chordoma is rarely detected before 
the age of $30 .{ }^{13}$ Our results showed a median age of 54.0 years for the chordoma of the mobile spine. In addition, the results showed higher male involvement in the older age group compared to women. The chordoma male predilection in our study is consistent with the previous literature reports as $2: 1 ., 7$ Our results showed an ASIR of 0.07 per million for mobile spine chordomas. Other studies $(0.08$ per 100000$)$ have reported a higher incidence for whole spine chordoma including the sacrococcygeal region. ${ }^{4}$

Osteosarcoma is high-grade malignant tumor composed of mesenchymal cells producing osteoid and immature bone. ${ }^{13}$ The incidence of osteosarcoma may be affected by race according to age at onset. There is a higher incidence rate among white population in the elder age group while African Americans have a higher incidence between the age of 25 to 29 years. ${ }^{3,12}$ By reviewing a series over 27 years, Bhatia et al reported that $69 \%$ of patients with spinal osteosarcoma represented primary spinal involvement and the rest were metastasis spread from other sites. ${ }^{23}$ Osteosarcoma accounts for 3\%-5\% of all malignancies of the spine. ${ }^{3}$ Osteosarcoma of the spine constitutes $0.6 \%$ to $3.2 \%$ of all diagnosed osteosarcomas. ${ }^{13}$ Of osteosarcomas of the spine, $30 \%$ occur in the sacrum and the rest affect the mobile spine. ${ }^{12,24}$ According to the NCDB, osteosarcoma is the least frequent primary osseous sarcoma of the spine among major sarcomas with only $13.1 \%$ of cases ${ }^{7}$; while $0.85 \%-3 \%$ of all osteosarcomas affect the spine. ${ }^{25}$ According to the INCR, osteosarcoma constitutes $12.3 \%$ of primary osseous mobile spine sarcomas. The ASIR of mobile spine osteosarcoma in Iran was 0.04 per million person-years. Osteosarcoma may occur from early childhood to old age ( 8 to 80 years); however, the peak incidence of this pathology is reported in the fourth decade in the literature which is higher than appendicular involvement. ${ }^{13,26-29}$ It is more common among adolescent and young adults; however, older patients in the seventh decade of life may encounter this pathology. ${ }^{12}$ The incidence of osteosarcoma in elderly patients is a consequence of long standing Paget's disease. ${ }^{3}$ Appendicular osteosarcoma affects the patients in a bimodal fashion, with the majority of cases occurring in the adolescent age group (interquartile 14-37). However, in the case of the osteosarcoma of the mobile spine, the median age is significantly higher and the average age of mobile spine osteosarcoma was 43 years (interquartile 23-61). In contrast with other studies, the INCR did not demonstrate a bimodal distribution for this pathology and the majority of new cases occurred in the age group of 1040 years including 20 cases (86\%). Like other sarcomas, there is a male predilection for osteosarcoma ${ }^{13}$ which is consistent with the results derived from the INCR.

In conclusion, primary osseous sarcoma of the mobile spine showed a significant male predilection. The median age of mobile spine sarcoma was 37.0 years which was different for each tumor type. Ewing sarcoma in Iran affects the mobile spine at slightly older ages while chondrosarcoma has a lower median age at the time of diagnosis compared to other studies. Myxoid chondrosarcoma is the most frequent subtype of the mobile spine chondrosarcomas excluding simply described chondrosarcoma. Chordoma affects men at an older age compared to women. In contrast with other studies, osteosarcoma of the spine affects young adults without affecting patients at older ages among the Iranian population.

This study represents the first epidemiologic investigation based on a national registry (INCR) for patients with primary osseous mobile spine sarcomas in Iran. While the INCR has many advantages, it is not without limitations. The methodological limitations of the study including sparse data and sparse-data bias should be borne in mind. ${ }^{30,31}$ In this registry, due to incomplete histopathology registration of the patients and lack of histology codes, histology evaluation was not possible. The INCR does not provide directly the survival data to enable the analysis and investigation of this valuable issue. Lack of data on surgical approach and treatment type was also a weakness of this study.

\section{Authors' Contributions}

AE: Coordination between university and ministry of health, data interpretation. MC designed the study, Conceived the experiment and revised the paper. MS: wrote the paper. MAL: statistical expert and data analyzing. AK: wrote the paper. AR: Coordination between University and Ministry of Health, data interpretation. AZ: Coordination between university and Ministry of Health, data interpretation. MEA: Coordination between University and Ministry of Health and conductor of tumor epidemiology in Iran.

\section{Conflict of Interest Disclosures}

The authors declare that they have no conflict of interest.

\section{Ethical Statement}

All procedures performed in studies involving human participants were in accordance with the ethical standards of the institutional and/or national research committee and with the 1964 Helsinki declaration and its later amendments or comparable ethical standards

Funding

There is no funding source.

\section{References}

1. Kim HJ, McLawhorn AS, Goldstein MJ, Boland PJ. Malignant osseous tumors of the pediatric spine. J Am Acad Orthop Surg. 2012;20(10):646-56. doi: 10.5435/ jaaos-20-10-646.

2. Wang VY, Potts M, Chou D. Sarcoma and the spinal column. Neurosurg Clin N Am. 2008;19(1):71-80. doi: 10.1016/j.nec.2007.10.001.

3. Ozturk AK, Gokaslan ZL, Wolinsky JP. Surgical treatment of sarcomas of the spine. Curr Treat Options Oncol. 2014;15(3):482-92. doi: 10.1007/s11864-014-0290-8.

4. Ropper AE, Cahill KS, Hanna JW, McCarthy EF, Gokaslan ZL, Chi JH. Primary vertebral tumors: a review of 
epidemiologic, histological and imaging findings, part II: locally aggressive and malignant tumors. Neurosurgery. 2012;70(1):211-9. doi: 10.1227/NEU.0b013e31822d5f17.

5. Groves ML, Zadnik PL, Kaloostian P, Sui J, Goodwin CR, Wolinsky JP, et al. Epidemiologic, functional, and oncologic outcome analysis of spinal sarcomas treated surgically at a single institution over 10 years. Spine J. 2015;15(1):110-4. doi: 10.1016/j.spinee.2014.07.005.

6. Mukherjee D, Chaichana KL, Gokaslan ZL, Aaronson O, Cheng JS, McGirt MJ. Survival of patients with malignant primary osseous spinal neoplasms: results from the Surveillance, Epidemiology, and End Results (SEER) database from 1973 to 2003. J Neurosurg Spine. 2011;14(2):143-50. doi: 10.3171/2010.10.spine10189.

7. Kerr DL, Dial BL, Lazarides AL, Catanzano AA, Lane WO, Blazer DG, 3rd, et al. Epidemiologic and survival trends in adult primary bone tumors of the spine. Spine J. 2019;19(12):1941-9. doi: 10.1016/j.spinee.2019.07.003.

8. Hesla AC, Bruland Ø S, Jebsen N, Styring E, Eriksson S, Tsagozis P. Ewing sarcoma of the mobile spine; predictive factors for survival, neurological function and local control. A Scandinavian sarcoma group study with a mean followup of 12 years. J Bone Oncol. 2019;14:100216. doi: 10.1016/j. jbo.2018.100216.

9. Akbari A, Khayamzadeh M, Salmanian R, Ghanbari Motlagh A, Roshandel G, Nouri M, et al. National cancer mortality-to-incidence ratio (MIR) in Iran (2005-2014). Int J Cancer Manag. 2019;12(6):e94145. doi: 10.5812/ ijcm.94145.

10. Ebrahimpour A, Chehrassan M, Sadighi M, Azizmohammad Looha M, Karimi A, Akbari A, et al. The survival and incidence rate of Ewing sarcoma; a national population-based study in Iran (2008-2015). Arch Bone Jt Surg. 2020;8(3):391-9. doi: 10.22038/abjs.2020.44095.2206.

11. Sobin L, Parkin DM. International Classification of Diseases for Oncology. Geneva: World Health Organization; 2000. p. 240.

12. Katonis P, Datsis G, Karantanas A, Kampouroglou A, Lianoudakis S, Licoudis S, et al. Spinal osteosarcoma. Clin Med Insights Oncol. 2013;7:199-208. doi: 10.4137/cmo. s10099.

13. Orguc S, Arkun R. Primary tumors of the spine. Semin Musculoskelet Radiol. 2014;18(3):280-99. doi: 10.1055/s0034-1375570.

14. Kelley SP, Ashford RU, Rao AS, Dickson RA. Primary bone tumours of the spine: a 42-year survey from the Leeds Regional Bone Tumour Registry. Eur Spine J. 2007;16(3):405-9. doi: 10.1007/s00586-006-0188-7.

15. Dahm PH, Bhattacharjee M, Huh W, Thapar V. Ewing's sarcoma of the cervical spine. J Pediatr Intensive Care. 2018;7(2):86-9. doi: 10.1055/s-0037-1606256.

16. Charest-Morin R, Dirks MS, Patel S, Boriani S, Luzzati A, Fehlings MG, et al. Ewing sarcoma of the spine: prognostic variables for survival and local control in surgically treated patients. Spine (Phila Pa 1976). 2018;43(9):622-9. doi: 10.1097/brs.0000000000002386.

17. Marco RA, Gentry JB, Rhines LD, Lewis VO, Wolinski JP, Jaffe N, et al. Ewing's sarcoma of the mobile spine. Spine
(Phila Pa 1976). 2005;30(7):769-73. doi: 10.1097/01. brs.0000157755.17502.d6.

18. Zhu C, Olson KA, Roth M, Geller DS, Gorlick RG, Gill J, et al. Provider views on the management of Ewing sarcoma of the spine and pelvis. J Surg Oncol. 2018;117(3):417-24. doi: 10.1002/jso.24860.

19. Boriani S, Bandiera S, Biagini R, Bacchini P, Boriani L, Cappuccio M, et al. Chordoma of the mobile spine: fifty years of experience. Spine (Phila Pa 1976). 2006;31(4):493503. doi: 10.1097/01.brs.0000200038.30869.27.

20. Arshi A, Sharim J, Park DY, Park HY, Bernthal NM, Yazdanshenas $\mathrm{H}$, et al. Chondrosarcoma of the osseous spine: an analysis of epidemiology, patient outcomes, and prognostic factors using the SEER registry from 1973 to 2012. Spine (Phila Pa 1976). 2017;42(9):644-52. doi: 10.1097/brs.0000000000001870.

21. Gietzen L, Pokorski P. Chondrosarcoma of the cervical spine. JAAPA. 2017;30(12):23-5. doi: 10.1097/01. JAA.0000525916.05473.da.

22. Chi JH, Bydon A, Hsieh P, Witham T, Wolinsky JP, Gokaslan ZL. Epidemiology and demographics for primary vertebral tumors. Neurosurg Clin N Am. 2008;19(1):1-4. doi: 10.1016/j.nec.2007.10.005.

23. Bhatia R, Beckles V, Fox Z, Tirabosco R, Rezajooi K, Casey AT. Osteosarcoma of the spine: dismal past, any hope for the future? Br J Neurosurg. 2014;28(4):495-502. doi: 10.3109/02688697.2013.869550.

24. Zils K, Bielack S, Wilhelm M, Werner M, Schwarz R, Windhager R, et al. Osteosarcoma of the mobile spine. Ann Oncol. 2013;24(8):2190-5. doi: 10.1093/annonc/mdt154.

25. Ozaki T, Flege S, Liljenqvist U, Hillmann A, Delling G, Salzer-Kuntschik M, et al. Osteosarcoma of the spine: experience of the Cooperative Osteosarcoma Study Group. Cancer. 2002;94(4):1069-77.

26. Dekutoski MB, Clarke MJ, Rose P, Luzzati A, Rhines LD, Varga PP, et al. Osteosarcoma of the spine: prognostic variables for local recurrence and overall survival, a multicenter ambispective study. J Neurosurg Spine. 2016;25(1):59-68. doi: 10.3171/2015.11.spine15870.

27. Feng D, Yang X, Liu T, Xiao J, Wu Z, Huang Q, et al. Osteosarcoma of the spine: surgical treatment and outcomes. World J Surg Oncol. 2013;11(1):89. doi: 10.1186/1477-7819-11-89.

28. Fisher CG, Saravanja DD, Dvorak MF, Rampersaud YR, Clarkson PW, Hurlbert J, et al. Surgical management of primary bone tumors of the spine: validation of an approach to enhance cure and reduce local recurrence. Spine (Phila Pa 1976). 2011;36(10):830-6. doi: 10.1097/ BRS.0b013e3181e502e5.

29. Schwab JH. Primary tumors of the spine. In: Orthopaedic Knowledge Update: Spine. Vol 5. Wolters Kluwer Health; 2018. p. 465-72.

30. Greenland S, Mansournia MA, Altman DG. Sparse data bias: a problem hiding in plain sight. BMJ. 2016;352:i1981. doi: 10.1136/bmj.i1981.

31. Agresti A. An Introduction to Categorical Data Analysis. Wiley Series in Probability and Statistics. Vol 423. USA: Wiley; 2006. 